\title{
Learning Induces Transient Upregulation of Brevican in the Auditory Cortex during Consolidation of Long-Term Memories
}

\author{
Hartmut Niekisch, ${ }^{1,2}$ ○ Julia Steinhardt, ${ }^{1}$ Julia Berghäuser, ${ }^{1}$ Sara Bertazzoni, ${ }^{1}{ }^{\circ}$ Erika Kaschinski, ${ }^{1}$ Jana Kasper, ${ }^{1}$

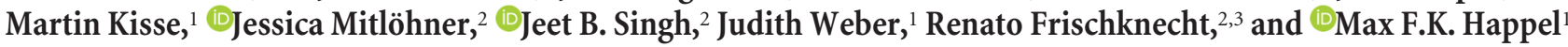 \\ Departments of ${ }^{1}$ Systems Physiology of Learning, ${ }^{2}$ Neurochemistry and Molecular Biology, Leibniz-Institute for Neurobiology, D-39118 Magdeburg, \\ Germany, and ${ }^{3}$ Department of Biology, Animal Physiology, Friedrich Alexander University Erlangen-Nuremberg, D-91058 Erlangen, Germany
}

It is a daily challenge for our brains to establish new memories via learning while providing stable storage of remote memories. In the adult vertebrate brain, bimodal regulation of the extracellular matrix (ECM) may regulate the delicate balance of learning-dependent plasticity and stable memory formation. Here, we trained adult male mice in a cortex-dependent auditory discrimination task and measured the abundance of ECM proteins brevican (BCN) and tenascin-R over the course of acquisition learning, consolidation, and long-term recall in two learning-relevant brain regions; the auditory cortex and hippocampus. Although early training led to a general downregulation of total ECM proteins, successful retrieval correlated with a region-specific and transient upregulation of BCN levels in the auditory cortex. No other parameter such as arousal or stress could account for the transient and region-specific BCN upregulation. This performance-dependent biphasic regulation of the ECM may assist transient plasticity to facilitate initial learning and subsequently promote the long-term consolidation of memory.

Key words: auditory cortex; behavior; extracellular matrix; hippocampus; learning; long-term memory

Significance Statement

The capacity to learn throughout life and at the same time guarantee lifelong storage and remote recall of established memories is a daily challenge. Emerging evidence suggests an important function of the extracellular matrix (ECM), a conglomerate of secreted proteins and polysaccharides in the adult vertebrate brain. We trained mice in an auditory long-term memory task and measured learning-related dynamic changes of the ECM protein brevican. Specifically, in the auditory cortex brevican is downregulated during initial learning and subsequently upregulated in exclusively those animals that have learned the task, suggesting a performance-dependent regulation in the service of memory consolidation and storage. Our data may provide novel therapeutic implications for several neuropsychiatric diseases involving dysregulation of the ECM.

\section{Introduction}

The brain has to provide plastic adaptability throughout life and concurrently guarantee lifelong storage of established memories.

\footnotetext{
Received Sept. 27, 2018; revised May 14, 2019; accepted May 14, 2019.

Author contributions: R.F. and M.F.K.H. designed research; H.N., J.S., J.B., S.B., E.K., J.K., and J.W. performed research; H.N., M.K., R.F., and M.F.K.H. analyzed data; J.M. and J.B.S. contributed unpublished reagents/analytic tools; R.F. and M.F.K.H. wrote the paper.

The work was supported by Grants from the Deutsche Forschungsgemeinschaft SFB779; B14 and B16), and by the Leibniz-Association (WGL). The funders had no role in study design, data collection and analysis, decision to publish, or preparation of the paper. We thank Dr. Marcus Jeschke for providing preliminary custom-written tools for behavioral analysis in MATLAB, Kathrin Hartung and Kathrin Ohl for technical assistance, and Katrina Deane for considerable proofreading of the paper.

The authors declare no competing financial interests.

Correspondence should be addressed to Renato Frischknecht at renato.frischknecht@fau.de or Max F. K. Happel at mhappel@lin-magdeburg.de.
}

Emerging evidence suggests that the extracellular matrix (ECM) plays a crucial role in balancing neuronal plasticity and stability (Pizzorusso et al., 2002; Happel et al., 2014; Bozzelli et al., 2018). The ECM in the mature brain is a conglomerate of proteoglycans and glycoproteins enwrapping neurons and synapses and reduces structural plasticity in adult vertebrates because of its repulsive properties (de Vivo et al., 2013). It appears in at least three forms: (1) a dense net-like form mainly found around parvalbuminpositive $(\mathrm{PV}+)$ inhibitory neurons called perineuronal nets (PNNs), (2) a cell-bound form, and (3) a "loose" form detected throughout the brain (Sorg et al., 2016). 
The attenuating effect of the ECM on plastic reorganizations associated with cognitive learning and memory consolidation is well documented. Mouse models deficient in tenascin-R (TNR) or brevican (BCN), both abundant ECM constituents of PNNs and loose ECM, show impaired forms of hippocampal long-term potentiation (Dityatev et al., 2010), altered function of PV+ interneurons (Favuzzi et al., 2017), and affected hippocampusdependent spatial or contextual acquisition learning (Morellini et al., 2010). Enzymatic depletion of the ECM by local injection of chondroitinase $\mathrm{ABC}$ (chABC) or hyaluronidase (HYase) into prefrontal cortex, hippocampus (CA), or auditory cortex (ACx) in wild-type mice impaired auditory contextual fear memory for 24-48 h (Hylin et al., 2013; Banerjee et al., 2017). Injections into perirhinal cortex prolonged object recognition memory (Romberg et al., 2013) and compensated impaired recall in an Alzheimer mouse model (Végh et al., 2014; Yang et al., 2015). The effect of enzymatic ECM removal depends on the timing and region of injection. For instance, ECM depletion in the amygdala did not affect acquisition of fear or drug memories, but promoted their subsequent extinction (Gogolla et al., 2009; Xue et al., 2014). Recently, we have shown that injection of HYase into the auditory cortex of Mongolian gerbils significantly improved the performance in an auditory cortex-dependent reversal learning task, while also not affecting acquisition learning (Happel et al., 2014). During states of weakened ECM, established memories might hence be more vulnerable to an activity-dependent change. Indeed, ECM proteins are targets of extracellular proteases, which may be activated during initial learning (Frischknecht et al., 2008; Dityatev et al., 2010). Thus, proteolytic cleavage of ECM proteins, such as BCN and their subsequent degradation, may reduce the integrity of the ECM and support structural plasticity. On the other hand, upregulation of ECM proteins at later learning phases may increase ECM density and promote synapse stabilization, which is necessary for long-term memory consolidation (Dudai, 2012). Thus, we hypothesized that endogenous regulation of ECM levels during initial learning and remote memory storage may assist both, learning-dependent plasticity and longterm consolidation.

To test this, we measured the abundance of the ECM proteins $\mathrm{BCN}$ and TNR during acquisition, consolidation, and long-term ( $>4$ weeks) memory recall during an auditory discrimination task in the auditory cortex and hippocampus of mice. Auditory training led to a general and immediate decrease of BCN abundance followed by a delayed and transient upregulation of the full-length protein during memory consolidation specifically in the auditory cortex as revealed by semiquantitative Western blots. Although upregulation of BCN in the ACx during consolidation was performance-dependent, $\mathrm{BCN}$ was not altered during long-term recall. Downregulation of BCN and TNR during initial learning in the $\mathrm{ACx}$ and $\mathrm{CA}$ was performance independent. This suggests that BCN and possibly other ECM molecules, such as TNR, are downregulated during initial training to facilitate learning-related network rearrangements and that transient, region-specific upregulation during retrieval is the necessary mediator for successful consolidation of recent memories for remote recall.

\section{Materials and Methods}

Ethics statement. Experiments were performed on adult male C57BL/6N mice (Mus musculus) at the age of 8-14 weeks (body weight: 27-36 g). Animals were obtained from Charles River Laboratories and housed in groups of three or four individuals per home cage on a $12 \mathrm{~h}$ light/dark cycle (light on at 6:00 a.m.). They had ad libitum access to standard laboratory chow and tap water. All experimental procedures were performed in accordance with the EU Council Directive 86/609/EEC and were approved and authorized by the local Committee for Ethics and Animal Research (Landesverwaltungsamt Halle, Germany) in accordance with the international NIH guidelines for animals in research.

Experimental design and statistical analysis. We assessed the learning behavior of animals in a cortex-dependent auditory learning paradigm (see next paragraph). We investigated the alteration of the ECM molecules BCN and TNR within auditory cortex over the course of four subsequent defined learning stages by using semiquantitative Western blot $(n=8$ each group; Fig. $1 A, B)$. Additionally, also a group of lowperforming animals $(n=7)$, a control group with pseudo-training by nonpaired tone and foot shock presentation $(n=8)$, and a naive animal group $(n=8)$ was investigated. Altogether, experiments were conducted on 55 animals.

For comparison of two samples, a paired Student's $t$ test was used. For statistical group evaluation, ANOVA (or Kruskal-Wallis) was performed. Tukey test for multiple comparisons or Bonferroni-correction were used for post hoc comparisons, respectively (GraphPad Prism 6). By correlation analyses, we tested the dependence of ECM protein regulation and behavioral parameters, like learning performance or number of foot shocks (RStudio). In all cases a significance level of $p<0.05$ was considered as statistically significant.

Behavioral experiments. Adult C57BL/6N mice were trained once per day to discriminate the direction of linear frequency modulated (FM) tones in a Go/NoGo two-way active avoidance shuttle-box paradigm (Wetzel et al., 1998; Ohl et al., 1999; Kähne et al., 2012; Happel et al., 2015; Reichenbach et al., 2015). The shuttle-box (TSE Technologies) was placed in an acoustically shielded chamber and contained two compartments separated by a hurdle (Fig. 1A, left). After 3 min of habituation to the training chamber without acoustical stimulation and foot shock, mice were trained to discriminate between a conditioned Go stimulus $(\mathrm{CS}+)$ and a conditioned NoGo stimulus (CS-) consisting of sequences ( $250 \mathrm{~ms}$ tone with $5 \mathrm{~ms}$ linear onset and offset ramps, $250 \mathrm{~ms}$ pause, $6 \mathrm{~s}$ duration) of a rising $(4-8 \mathrm{kHz})$ and of a falling $(8-4 \mathrm{kHz})$ frequencymodulated tone. The unconditioned stimulus (US) was a mild foot shock (started with $200 \mu \mathrm{A}$ ) administered through a metal floor grid. Current applied for foot shock was adapted for each animal individually to elicit comparable response strengths for the escape behavior (Fig. 1C; Happel et al., 2015). The conditioned Go response (CR+) was defined as the first change of shuttle-box compartments by crossing the hurdle within an observation window of $6 \mathrm{~s}$ after the onset of the CS. Staying in the compartment was defined as NoGo conditioned response (CR-). A CR+ during the observation window in response to a CS+ or CS- was counted as a hit or false alarm, respectively. No shuttling in the observation window in response to a CS+ or CS- was counted as a miss or correct rejection, respectively. The foot shock is always turned off when animals changed compartments in response to the US (escape response). Other compartment changes were classified as intertrial shuttles (ITS). Each daily session consisted of 60 trials, that is, 30 presentations of each $\mathrm{CS}+$ and CS - in a pseudorandomized order (mean intertrial interval: $20 \pm 5$ s). Stimuli were delivered through calibrated loudspeakers at $\sim 75$ dB SPL.

We calculated conditioned response learning curves for CS + and CSseparately $($ hit rate $=$ hits/number of CS + trials; false alarm rates $=$ false alarms/number of CS - trials; Fig. 1A, right). We further used the sensitivity index $d^{\prime}$, which allows to assess the behavioral sensitivity independent of experimental conditions biasing the response of the animal based on signal detection theory (Deliano et al., 2009). For $d^{\prime}$ analysis, $z$-scores of corresponding hit and false-alarm rates were derived from the inverses of a standardized normal distribution function and divided $\left(d^{\prime}=z_{\text {hits }}\right.$ $\mathrm{Z}_{\mathrm{false}}$ alarms $)$. Values of $d^{\prime}=1.0$ correspond to a signal discrimination strength of 1 SD above noise, which we defined as threshold criterion for robust auditory discrimination (Happel et al., 2015).

Based on CR rates and $d^{\prime}$, the learning performance was scored into training stages (Fig. 2). Within the first one or two sessions, animals reduced their escape latencies to $<1 \mathrm{~s}$ after the onset of the US (Fig. 1C). Next, subjects shuttled in response to the CS before US onset to avoid the mild foot shock, which is referred to as avoidance learning (AV; $n=8$ ). 
A

2-way shuttle-box Go/NoGo paradigm
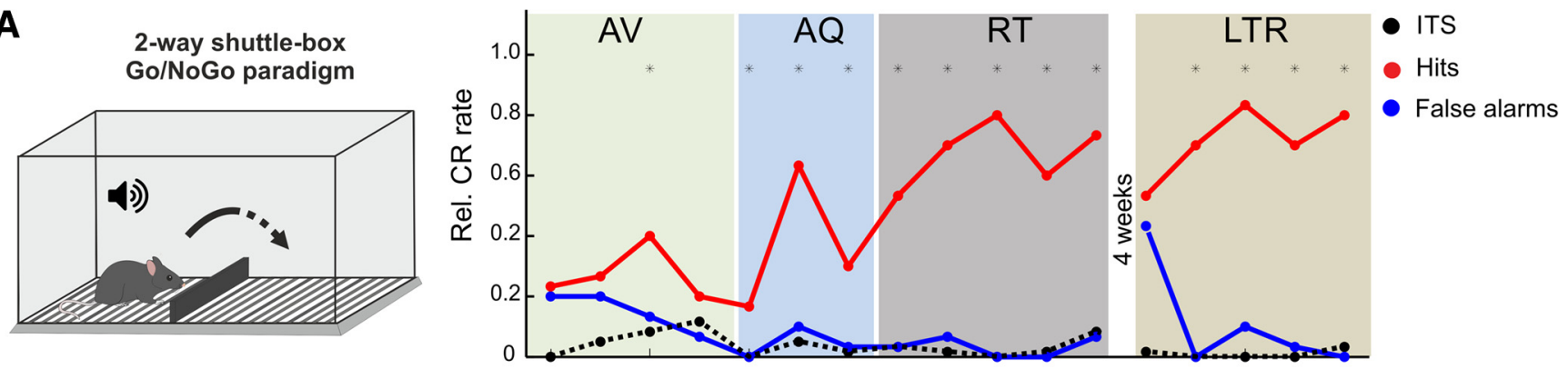

B

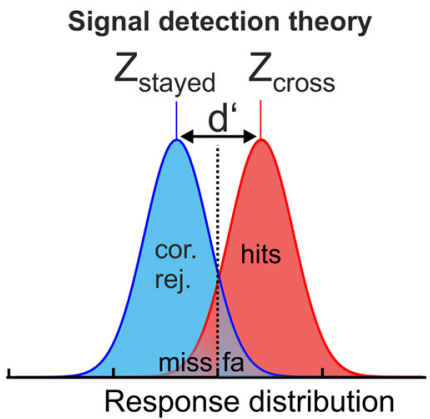

C



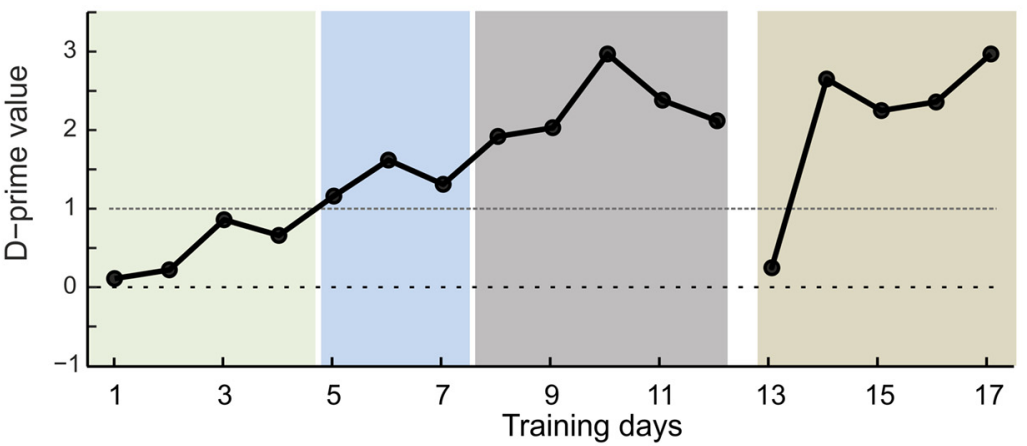

Histogram

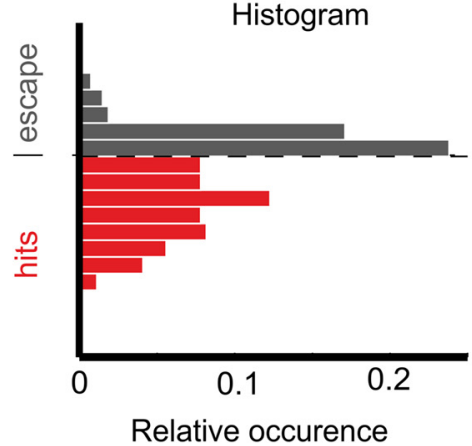

Figure 1. Cortex-dependent auditory learning in mice trained in a shuttle-box paradigm. A, Left, Animals are trained in a two-compartmental shuttle-box. Right, Mean CRs are measured for Go (red) and NoGo trials (blue) and termed hits and false alarms, respectively (Happel et al., 2015). Dashed black line indicates mean ITS. Subjects pass through consecutive learning stages of early AV, $A Q$, and RT of the learned contingencies. After 4 weeks of retention interval, we tested their LTR performance. All groups contain a number of $n=8$. Of all trained animals, seven subjects did not yield a significant discrimination (LPs). *Asterisks mark significant differences between hits and false alarms revealed by paired Student's $t$ test $(p<0.05)$. B, Left, Based on signal detection theory, discrimination performance was measured as $d^{\prime}$ per training day. Right, Corresponding $d^{\prime}$ learning curve based on CR rates shown in $A$. C, Response latencies during CS+ trials are plotted for individual trials over all training sessions. Black crosses represent median (horizontal) and SD of escape latencies $>6 \mathrm{~s}$ within a session. Escape latencies decreased below a range of $1 \mathrm{~s}$ after US onset after individual increase of the foot shock. Response latencies $<6 \mathrm{~s}$ correspond to successful hit responses. Histograms (right) of response latencies show a bimodal distribution corresponding to hit responses $(<6 s)$ and escape responses $(6-8 \mathrm{~s})$.

This was quantified by a ${d^{\prime}}_{\mathrm{AV}}>1.0$ calculated for CS + trials against the ITS as reference of spontaneous shuttling $\left(d^{\prime}{ }_{\mathrm{AV}}=\mathrm{z}_{\mathrm{hits}} / \mathrm{z}_{\mathrm{ITS}}\right)$. In a next step, animals reduced the number of false alarms and thereby established a successful discrimination strategy. If animals reached the threshold criterion of $d^{\prime}>1.0$ for three consecutive sessions, we referred to as the acquisition stage during which animals showed the steepest learning progress (AQ; $n=8$ ). Animals were hence killed $48 \mathrm{~h}$ after their first significant discrimination performance. A group of animals that quickly accelerated acquisition was trained until they performed 5 training days or longer above $d^{\prime}>1.0$ and hence were in the retrieval phase of consolidated memory recall (RT; $n=8)$. Another group that has reached this retrieval criterion after 12 training days has been kept in the home cage over a retention interval of 4 weeks. Then, long-term recall (LTR; $n=8$ ) performance was tested over additional five training sessions. For each training group (AV, AQ, RT, LTR) we trained eight animals that were killed directly after reaching the corresponding training stage. Brains were removed for protein biochemistry. In the entire training set, we had a group of seven mice that only reached the avoidance criterion, but failed to discriminate the two FM signals. Hence, animals of this group did not acquire the acquisition or retrieval stage of the task within the first 10 training sessions. This group is henceforth referred to as the lowperforming group (LP; $n=7$ ). This group experienced the highest levels of foot shocks per session without a considerable decrease of the shock rate over the 10 training sessions (Fig. 2D). To control against general effects of tone or shock presentation, another control group of eight animals received six sessions with unpaired and randomized tones and foot shocks (30 shocks per session) and subsequently four sessions without foot shocks. Altogether, this group received the same amount of tones and foot shocks over 10 training sessions, as in the retrieval group. On average, animals in the retrieval group received 180 foot shocks over 10 training sessions with lower numbers in later sessions because of higher performance. The control group hence tests for the specificity of auditory association learning and controls against potential effects of relief of potential arousal/stress over the training procedure (see Fig. 5).

Semiquantitative Western blot and immunofluorescence detection. Animals from each learning stage group were anesthetized using isoflurane (4\%) and killed directly after the last training session. For tissue preparation, brains were removed and bilateral hippocampus and auditory 
A
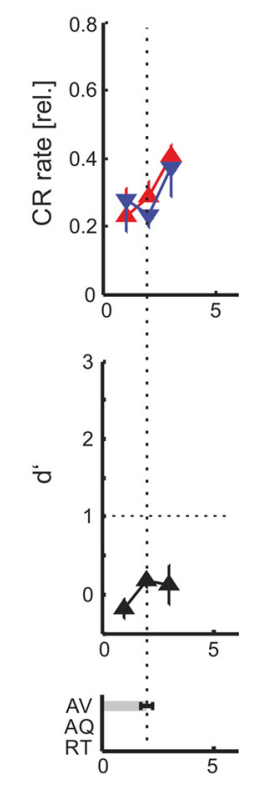

Training days
$A Q$ (acquisition)

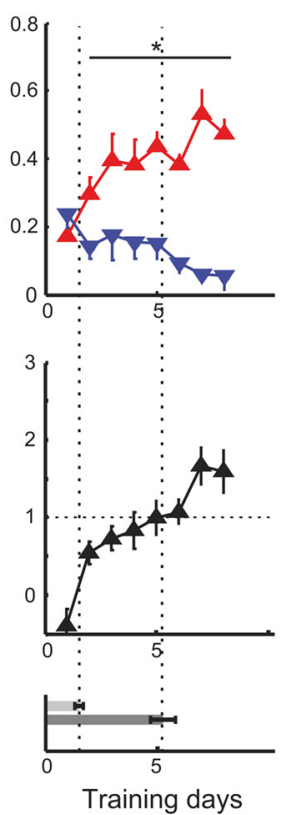

RT (retrieval)
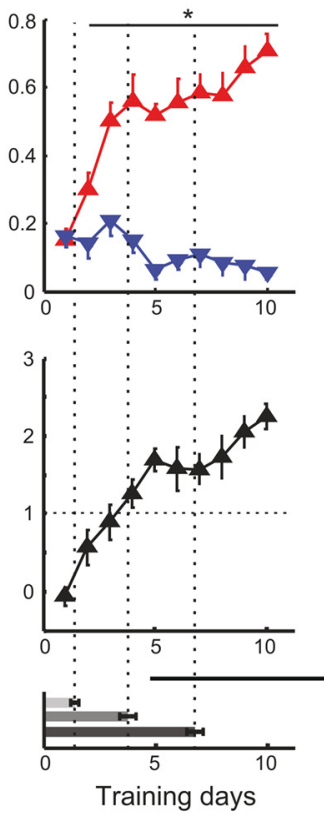

LP (low performer)
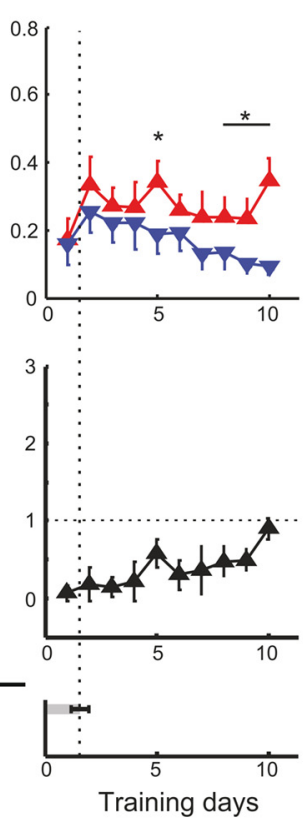

LTR (long-term recall)

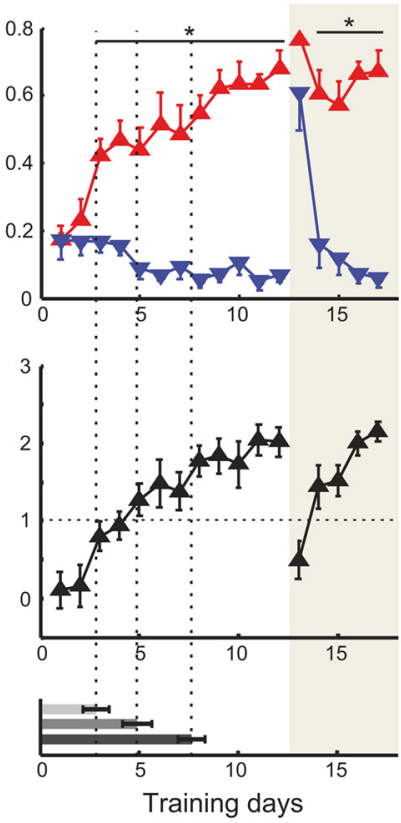

Go NoGo
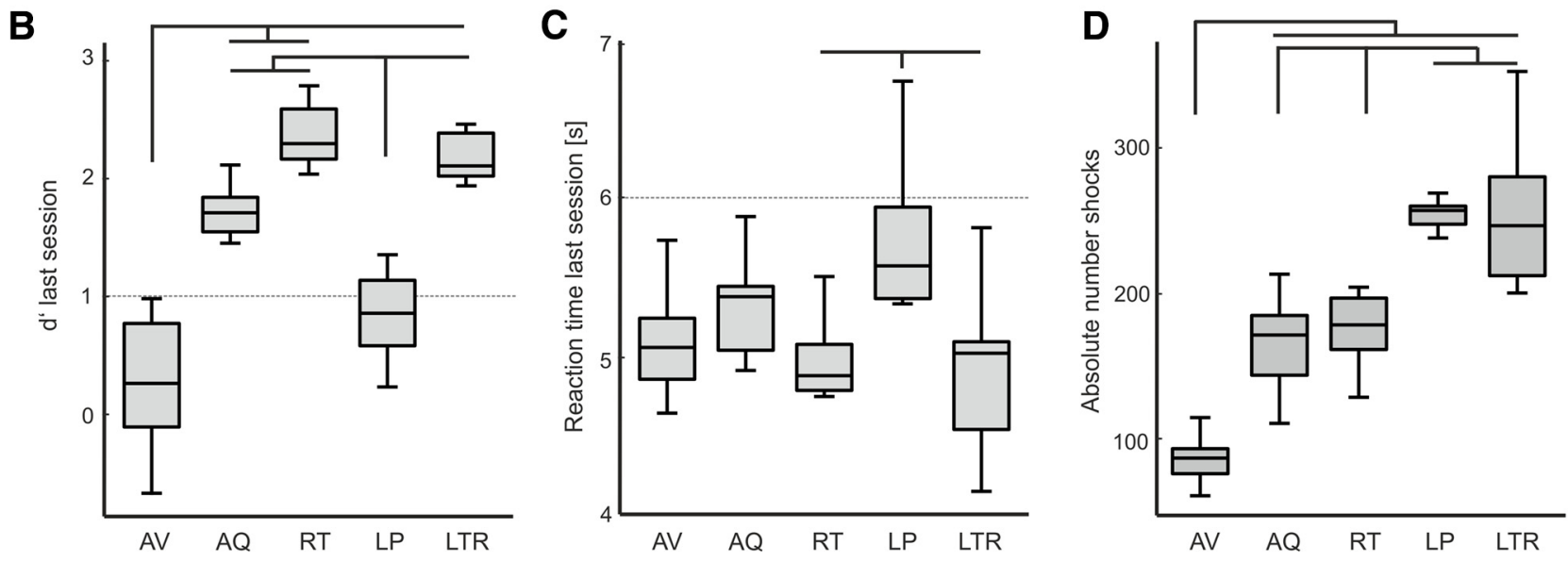

Figure 2. Learning curves (LCS) of animals trained to reach distinct learning stages for subsequent Western blot analysis. $A$, Top, CR curves (mean \pm SEM) of adult male C57BL/6N mice ( $n=39$; $n=8$ in each group; $\mathrm{LP}=7$ ) in a Go/NoGo shuttle-box-paradigm during FM discrimination (4-8vs $8-4 \mathrm{kHz}$ ). Relative hit rate (red) is plotted against relative false alarm rate (blue) across training days (1 session per day/60 trials). Middle, Sensitivity index $d^{\prime}$ (mean \pm SEM) was used to define distinct learning stages from avoidance, acquisition, retrieval, and a group of $L P$ animals. Another set that reached the retrieval-stage was trained for another $5 \mathrm{~d}$ after a retention interval of 4 weeks to test their LTR performance. Bottom, Averaged number of sessions of each group to reach the respective learning stages (mean \pm SEM) did not show significant differences across groups (Table $1 A$ ). Corresponding dotted lines in top indicate learning stage transitions. *Paired Student'st test, $p<0.05$. Abbreviations for training stages will be used in following figures. $B$, Group comparison of mean $d^{\prime}$ of last sessions across trained animal groups revealed significantly different performance levels between the AV and LP groups compared against AQ, RT, and LTR (Kruskal-Wallis; $p<0.001$ ). $C$, Mean reaction times across all groups revealed significantly longest reaction times in low performing animals (Kruskal-Wallis; $p=0.012$ ). D. Absolute number of shocks received over the training paradigm significantly increased with number of trained sessions and low performance (Kruskal-Wallis; $p<0.001$ ). All indicated bars are based on Bonferroni-corrected significance levels. Box plots represent median (bar) and interquartile range, and whiskers represent full range of data.

cortex were localized and surgically removed on the basis of their stereotaxic coordinates (Kähne et al., 2012; CA on the basis of its structure and ACx: bregma -2.06 to -3.4 , size: rostrocaudal $2 \mathrm{~mm}$, dorsoventral 1.3 $\mathrm{mm})$. Tissue was dissected and immediately frozen in liquid nitrogen and stored at $-80^{\circ} \mathrm{C}$. Probes were then thawed on ice and the ECM was extracted for $45 \mathrm{~min}$ at $37^{\circ} \mathrm{C}$ using chondroitinase extraction buffer $(0.1$ M Tris-HC 0.03 M sodium acetate, including protease inhibitor ROCHE complete ULTRA Tablets EDTA-free, $\mathrm{pH}$ 8.0) containing $1 \mu \mathrm{l}$ chondroitinase ABC (EC 4.2.2.4, Sigma-Aldrich; $0.1 \mathrm{U}$ ) per $800 \mu \mathrm{l}$ buffer at a ratio $1: 10(\mathrm{w} / \mathrm{v})$. Subsequently, probes were centrifuged at $4^{\circ} \mathrm{C}, 12,000$ $\mathrm{U} / \mathrm{min}$ (Eppendorf Microcentrifuge, $5424 \mathrm{R}$ ). Pellets and supernatants were mixed with Laemmli buffer and analyzed by SDS-PAGE and Western blot using Tris-Glycin 5-20\% gradient gels containing 2,2,2trichloroethanol (TCE) to visualize and quantify total protein amount
(Ladner et al., 2004). Each fraction was loaded twice and gels were blotted on polyvinylidene difluoride (Millipore) membranes using the Mighty Small Transfer Tank system (Hoefer). Blots were then rinsed with Trisbuffered salt solution containing $0.1 \%$ Tween 20 (TBS-T) and blocked for $45 \mathrm{~min}$ in TBS-T containing $5 \%$ bovine serum albumin. Blots were then incubated with primary antibody solution ( $\mathrm{ms}$ anti- BCN, 1:1000; BD Transduction Laboratories, catalog \#610894; goat anti-TNR 1:200, Santa Cruz Biotechnology, catalog \#Sc-9875; in TBS-T). Subsequently, they were incubated with corresponding fluorescence coupled secondary antibody (donkey anti mouse AlexaFluor 680, donkey anti-goat AlexaFluor 800; Li-COR). Blots were scanned using the Li-COR system (Odyssey) and signals quantified using ImageJ. Signal intensity was normalized against a standard sample (total brain homogenate) for each blot and corrected for protein load determined by UV-visualization of total pro- 
tein labeled with TCE (Ladner et al., 2004). Within each blot, fluorescence intensity for each protein band was normalized to the naive sample.

\section{Results}

We trained mice in an auditory cortex-dependent discrimination learning and LTR paradigm in a shuttle-box (see Materials and Methods). We investigated the abundance of BCN and TNR in auditory cortex and hippocampus at different learning phases and LTR by semiquantitative Western blotting.

\section{Classification of learning stages in a cortex-dependent auditory discrimination task}

In the first part of this study, individual groups of animals (total $n=39$ ) were trained to discriminate two frequency-modulated tones as conditioned stimuli based on their modulation direction (rising $4-8 \mathrm{kHz}$ vs falling $8-4 \mathrm{kHz} ; 75 \mathrm{~dB}$ SPL) in an aversive two-way Go/NoGo shuttle-box paradigm (Fig. 1A; Wetzel et al., 1998). This learning process has been demonstrated to depend on learning-related plasticity in auditory cortex (Ohl et al., 1999; Ohl and Scheich, 2005). We classified distinct learning stages based on conditioned response rates and learning curves based on the sensitivity index $d^{\prime}$ (see Materials and Methods) within individual subjects (Fig. $1 A, B$ ). A first group was trained until animals showed significantly increased hit responses compared with spontaneous intertrial shuttles for 2 consecutive days avoiding foot shock punishment in Go trials (Fig. 2A). In this early learning stage animals generally increased shuttling in response to both conditioned stimuli. Hit and false alarm rates did not significantly differ yielding a $d^{\prime}<1.0$. Therefore, we refer to this stage as AV ( $n=8$; cf. Stark and Scheich, 1997). Animals needed $2.0 \pm 0.10$ sessions to reach the avoidance criterion (Fig. $2 A$, bottom). A next group of animals was trained until they started to significantly discriminate between $\mathrm{CS}+$ and $\mathrm{CS}-$ based on a threshold criterion of $d^{\prime}>1.0$ for three consecutive sessions, which we refer to as the AQ $(n=8)$. In this group, animals had their first session with a significant discrimination on average after $5.25 \pm 0.56$ sessions and were killed for biochemical analysis $48 \mathrm{~h}$ later. Another set of animals acquired discrimination and showed a stable performance over five or more consecutive sessions above $d^{\prime}>1.0$ within the first 10 training days referred to as the RT group $(n=8)$. The retrieval criterion in this group was reached after $6.8 \pm 0.38$ sessions. Hence, after $10 \mathrm{~d}$ animals of the RT group have reached a consolidation phase of the training procedure. Animals that failed to reach the AQ and RT criterion because of unsuccessful discrimination even after $10 \mathrm{~d}$ were sorted into a group of LP animals $(n=7)$. This group only reached the avoidance criterion, but did not establish a significant discrimination of the both CS $\left(d^{\prime}<1.0\right)$. Across the entire training set, 7 of the total 39 trained animals were classified as LPs. A last group of learners, which had reached the RT criterion within 12 training days was trained again after a retention interval of 4 weeks. During LTR $(n=8)$ all animals showed initially an avoidance strategy (shuttling to both CS without discrimination) within the first LTR session (Fig. $2 A$, right). In following sessions, animals quickly reduced their false alarm rate and showed a successful recall of the correct discrimination behavior yielding $d^{\prime}>1.0$.

All groups reached the individual learning stage criteria within a comparable amount of sessions (one-way ANOVAs of sessions needed to reach a respective learning stage revealed no significant differences between all groups, $p<0.05$; Table $1 A$ ). Chosen criteria of learning stages led to considerable differences in perfor-
Table 1. Statistical analysis of behavioral and biochemistry data of animals

\begin{tabular}{|c|c|c|}
\hline Factor & Fvalue & $p$ value \\
\hline \multicolumn{3}{|c|}{ A. First stage transition between trained groups of animals (one-way ANOVA, $\alpha^{*}=0.05$ ); Fig. $2 A$} \\
\hline AV session & $F_{(4,31)}=2.323$ & $p=0.0768$ \\
\hline AQ session & $F_{(2,21)}=1.832$ & $p=0.1847$ \\
\hline RT Session & Unpaired Student's $t$ test & $p=0.2761$ \\
\hline \multicolumn{3}{|c|}{ B. Quantification absolute values of BCN ECM-fraction (one-way ANOVA, $\alpha^{*}=0.05$ ); Fig. 3C, } \\
\hline $145 \mathrm{kDa} B C N \mathrm{ACx}$ & $F_{(5,41)}=3.342$ & $p=0.0127$ \\
\hline $55 \mathrm{kDa} B C N \mathrm{ACX}$ & $F_{(5,41)}=3.434$ & $p=0.0111$ \\
\hline 145 kDa BCN CA & $F_{(5,41)}=0.3497$ & $p=0.8795$ \\
\hline $55 \mathrm{kDa}$ BCN CA & $F_{(5,41)}^{(1,+11)}=1.062$ & $p=0.3954$ \\
\hline \multicolumn{3}{|c|}{ C. Quantification absolute values of TNR ECM-fraction (one-way ANOVA, $\alpha^{*}=0.05$ ); Fig. $4 C, L$} \\
\hline Full-length TNR ACX & $F_{(5,41)}=2.085$ & $p=0.0868$ \\
\hline Full-length TNR CA & $F_{(5,41)}=1.818$ & $p=0.1306$ \\
\hline \multicolumn{3}{|c|}{ D. Quantification absolute values of $B C N$ cellularfraction (one-way ANOVA, $\alpha^{*}=0.05$ ); Fig. $3 C, D$} \\
\hline $145 \mathrm{kDa} B C N \mathrm{ACx}$ & $F_{(5,41)}=1.758$ & $p=0.1433$ \\
\hline $55 \mathrm{kDa} B C N \mathrm{ACx}$ & $F_{(5,41)}=15.79$ & $p<0.0001$ \\
\hline $145 \mathrm{kDa}$ BCN CA & $F_{(5,41)}^{(3,41)}=1.19$ & $p=0.3309$ \\
\hline $55 \mathrm{kDa} B C N C A$ & $F_{(5,41)}^{(1,41)}=5.221$ & $p=0.0008$ \\
\hline \multicolumn{3}{|c|}{ E. Quantification absolute values of TNR cellular fraction (one way ANOVA, $\alpha^{*}=0.05$ ); Fig. $4 C, D$} \\
\hline Full-length TNR $A C x$ & $F_{(5,41)}=1.668$ & $p=0,1640$ \\
\hline Full-length TNR CA & $F_{(5,41)}^{(0,41)}=1.422$ & $p=0.2367$ \\
\hline \multicolumn{3}{|c|}{ F. Quantification total levels of BCN (one-way ANOVA, $\alpha^{*}=0.05$ ); Fig. $7 A, B$} \\
\hline Sum of $55 \mathrm{kDa} B C N A C x$ & $F_{(5,41)}=14.6$ & $p<0.0001$ \\
\hline Sum of $145 \mathrm{kDa} B C N A C x$ & $F_{(5,41)}=5.567$ & $p=0.0005$ \\
\hline Sum of $55 \mathrm{kDa} B C N C A$ & $F_{(5,41)}=6.377$ & $p=0.0002$ \\
\hline Sum of $145 \mathrm{kDa} B C N \mathrm{CA}$ & $F_{(5,41)}^{(3,41)}=1.632$ & $p=0.1733$ \\
\hline \multicolumn{3}{|c|}{ G. Quantification total levels of TNR (one-way ANOVA, $\alpha^{*}=0.05$ ); Fig. $7 A, B$} \\
\hline Sum of full-length TNR $A C x$ & $F_{(5,41)}=2.025$ & $p=0.0952$ \\
\hline Sum of full-length TNR CA & $F_{(5,41)}=3.773$ & $p=0.0067$ \\
\hline
\end{tabular}

All tests were based on significance level of $\alpha^{*}=0.05$; for ANOVA post hoc analysis Tukey's tests of multiple comparisons was used. Corresponding figures for tests are indicated.

mance across training groups revealed by a significantly lower mean $d^{\prime}$ in the last session of the AV and LP group compared with all other training groups (Fig. $2 B$ ). Furthermore, with increasing performance reaction times in avoidance learning generally decrease (cf. Logan, 1992). Animals showed a corresponding trend of shortest reaction times in the retrieval and LTR groups and were significantly longer in LP animals (Fig. 2C). As an indicator of overall aversive load, and hence potential arousal, absolute numbers of shocks differed between groups and significantly increased with length of training and task failure (Fig. 2D). To test for effects of general arousal and specificity of associative learning, a group of control animals received a comparable amount of tones and shocks over 10 daily sessions as animals in the retrieval group (see Fig. 5). Brains of each animal were removed directly after the last training session and used for further Western blot analyses.

\section{Complementary learning-dependent alterations of full-length} and cleaved $\mathrm{BCN}$ are specific for auditory cortex

We collected samples from bilateral auditory cortex and hippocampus of animals trained to the aforementioned learning stages and quantified the abundance of BCN and TNR as representative proteins of the ECM on semiquantitative Western blots ( $n=39$ trained, $n=8$ naive, $n=8$ non-associative control). Although the three forms of the ECM have a similar molecular composition, they differ in their biochemical properties. Although the loose ECM is highly soluble, PNNs and cell-bound ECM need denaturing conditions to be extracted from tissue (Deepa et al., 2006). We treated dissected tissue with chABC, which releases most of the ECM components to the supernatant. Subsequently samples were centrifugated to separately analyze soluble and cellular components corresponding to loose and 
A

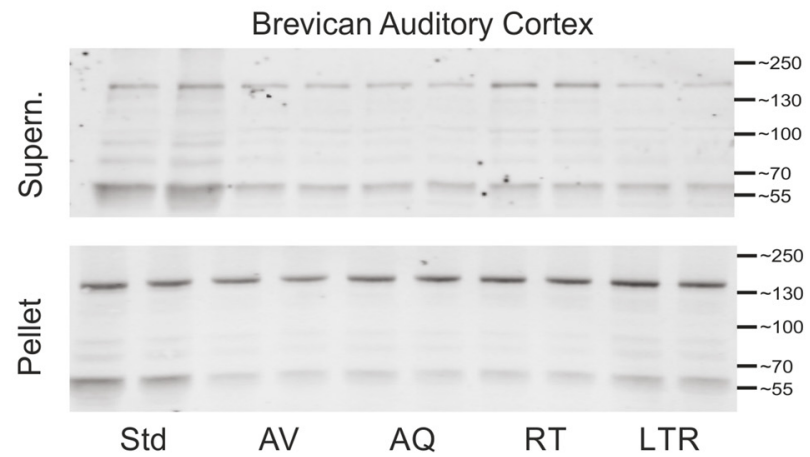

C
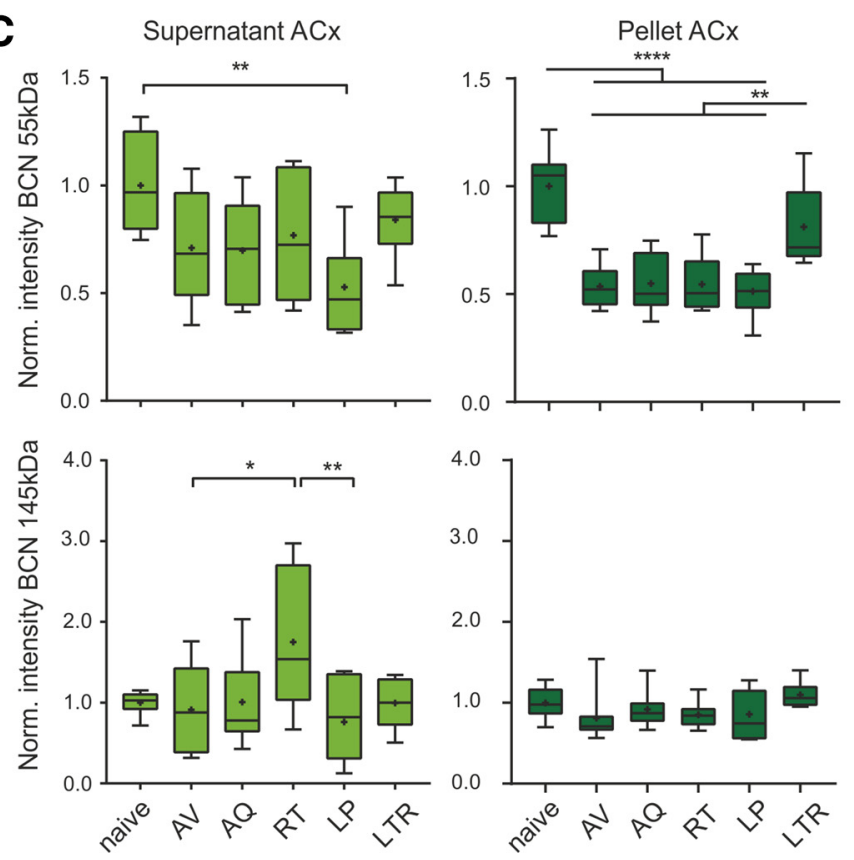

B
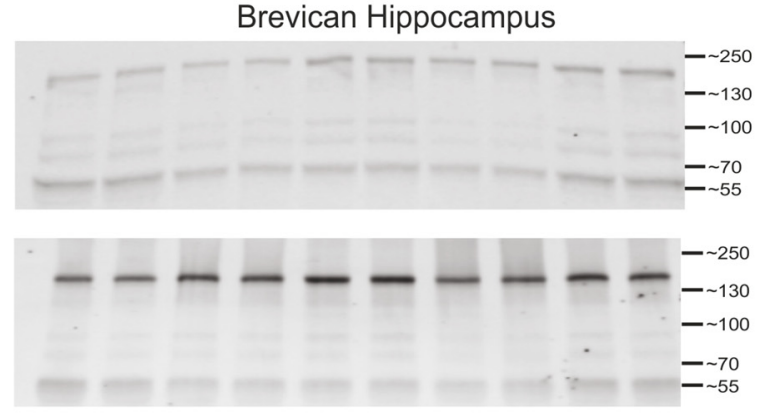

$\begin{array}{lllll}\text { Std } & \text { AV } & \text { AQ } & \text { RT } & \text { LTR }\end{array}$

Pellet CA
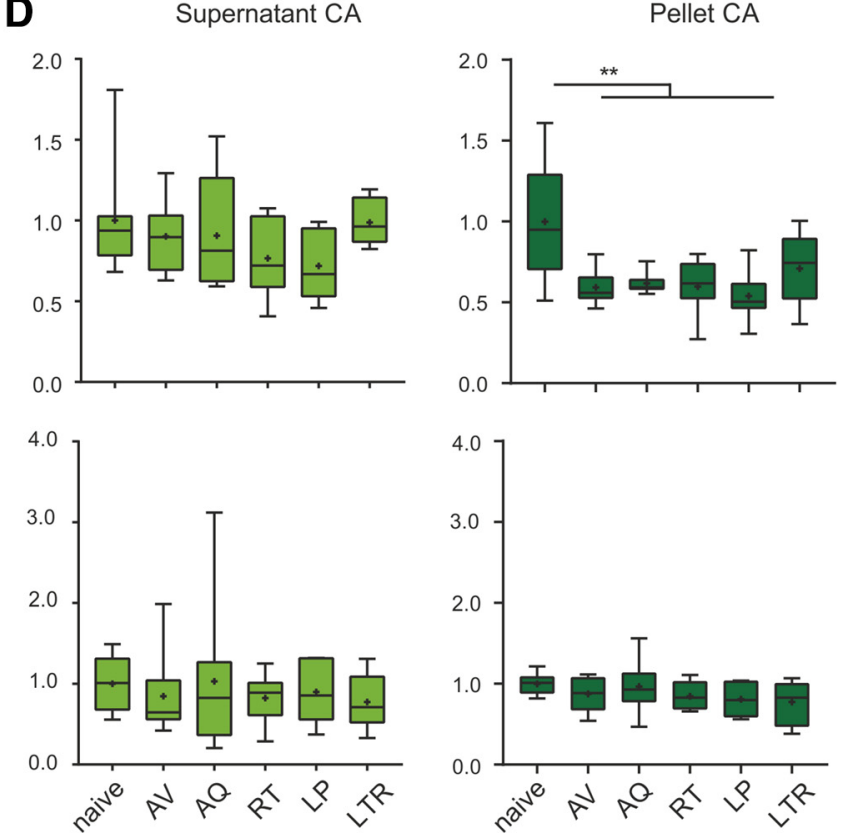

Figure 3. Semiquantitative Western blot analysis of learning-induced changes of BCN levels in auditory cortex and hippocampus. $\boldsymbol{A}, \boldsymbol{B}$, Top, Representative Western blot example treated with mouse anti-BCN antibody directed against the N-terminus detected the full-length BCN of $145 \mathrm{kDa}$ and the $55 \mathrm{kDa}$-terminal fragment within the cellular and the ECM fraction in auditory cortex $(\boldsymbol{A})$ and hippocampus $(\boldsymbol{B})$. The two lanes for each group correspond to the same sample, as probes were loaded twice. Quantification of signal intensity of BCN ( $55 \mathrm{kDa}$, $145 \mathrm{kDa}$ ) in the extracellular fraction (top) and cellular fraction (bottom). C, Across training groups, full-length BCN showed an extracellular increase exclusively in the RT group (bottom left). The $55 \mathrm{kDa}$ proteolytic fragment otherwise showed a general decrease, with significant difference in low-performers. In the cellular fraction, full-length proteins showed no significant changes across all training groups, whereas the $55 \mathrm{kDa}$ BCN showed consistently significantly lower levels in all training groups except of the LTR group. D, In hippocampus, significant reduction was only found in the pellet fraction of the 55 kDa BCN fragment in all training groups. Other protein levels in hippocampus did not show significant changes. * Indicates significant one-way ANOVA and post hoc Tukey tests of multiple comparisons between groups (Table $1 B-D$ ). Box plots represent median (bar) and interquartile range, and bars represent full range of data. Plus within each bar represents mean value.

PNN/cellular ECM, respectively (Fig. $3 A, B$ ). BCN appears as 145 $\mathrm{kDa}$ full-length and $55 \mathrm{kDa} \mathrm{N}$-terminal proteolytic fragment, which were analyzed separately (Fig. $3 A$ ).

Analysis of the ECM fraction (supernatant) of ACx showed differential, learning-stage-dependent changes of the $145 \mathrm{kDa}$ fulllength and $55 \mathrm{kDa}$ proteolytic fragment of $\mathrm{BCN}$ compared with a naive control group (Fig. 3C). Behavioral training led to a decrease of the $55 \mathrm{kDa}$ fragment of $\mathrm{BCN}$ in all trained animals, but reached statistical significance only in animals that failed to discriminate the two CS (Table $1 B$ ). The $145 \mathrm{kDa}$ full-length $\mathrm{BCN}$ showed no change during early training in the soluble fraction, but was significantly upregulated in the retrieval group revealed by a significant one-way ANOVA and post hoc Tukey tests of multiple comparisons (Table $1 B)$. This upregulation with successful consolidation of the recent auditory discrimination memory was found to be transient, as levels recovered back to baseline in the LTR group.

The cellular fraction (pellet) of auditory cortex samples is enriched in PNN and cellular ECM (Deepa et al., 2006). In this fraction the $145 \mathrm{kDa}$ full-length $\mathrm{BCN}$ showed no significant regulation during initial training compared with naive animals in (Fig. 3C). In contrast, the $55 \mathrm{kDa}$ proteolytic fragment of $\mathrm{BCN}$ was significantly less abundant in all training groups independent of performance except for the LTR group. During LTR, animals showed a restored amount of the $55 \mathrm{kDa}$ fragment comparable to levels in naive animals (Fig. $3 C$; Table $1 D$ ).

The auditory cortex undergoes plastic reorganizations during the task under investigation (Ohl et al., 1999). To test for general training-derived alterations, for instance reflecting contextual learning, in addition to region-specific changes within auditory cortex, we further analyzed changes of $\mathrm{BCN}$ in the hippocampus. Indeed, also in the hippocampus the $55 \mathrm{kDa} \mathrm{BCN}$ fragment in the pellet fraction was significantly reduced in all training groups (Fig. $3 B, D$; Table $1 D$ ). The soluble fraction showed only a minor decrease of the $55 \mathrm{kDa} \mathrm{BCN}$, but not significantly different from naive controls. Further, full-length BCN showed only a nonsignificant tendency for downregulation in the soluble and the cel- 
A



C

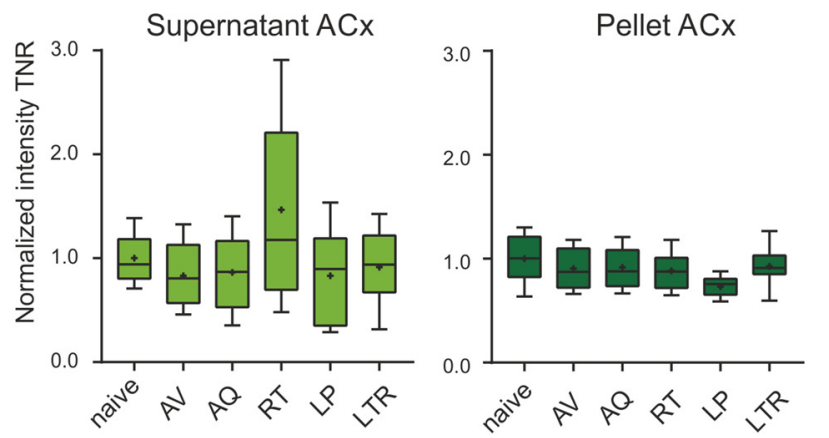

B

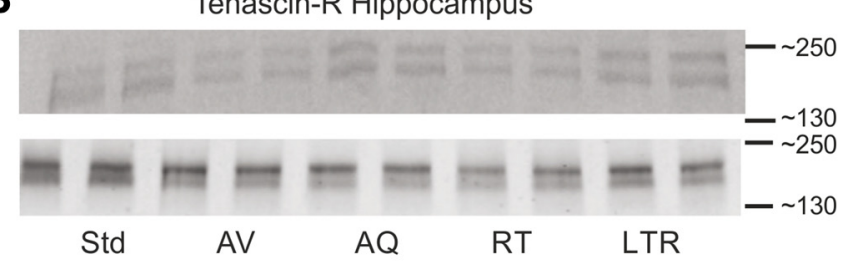

D

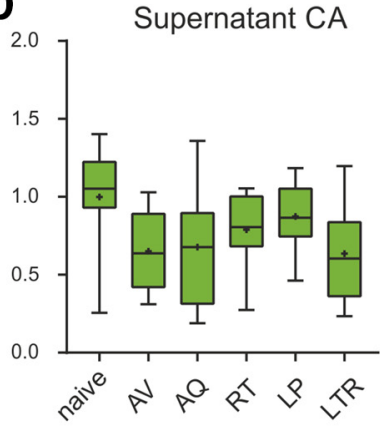

Pellet CA

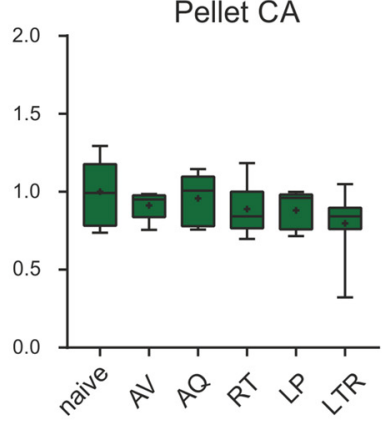

Figure 4. Semiquantitative Western blot analysis of learning-induced changes of TNR levels in auditory cortex and hippocampus. $A, B$, Top, Representative Western blot example treated with antibody against TNR in auditory cortex $(\boldsymbol{A})$ and hippocampus $(\boldsymbol{B})$. The two lanes for each group correspond to the same sample, as probes were loaded twice. Quantification of signal intensity of TNR in the extracellular fraction (top) and cellular fraction (bottom). C, Consistently with BCN, TNR also showed an extracellular increase exclusively in the RT group, but without significant difference to the naive group (left). In the cellular fraction of auditory cortex ( $\boldsymbol{C}$, right) and hippocampus ( $\boldsymbol{D}$, right) TNR showed no significant changes across all training groups. The supernatant fraction of TNR in hippocampus did also showed nonsignificant trends toward a reduction across training groups ( $\boldsymbol{D}$, left). A one-way ANOVA with multiple comparisons between groups revealed no significant differences (Table 1C $E$ ). Box plots represent median (bar) and interquartile range, and bars represent full range of data. Plus within each bar represents mean value.

lular fraction within any training phase compared with naive controls (Table $1 B-D$ ).

In addition, we investigated regulation of TNR, a binding partner of BCN (Fig. 4). In auditory cortex, we found a similar trend of decreased abundance of TNR with onset of training in the cellular fraction. In the soluble fraction, we observed a transient increase of TNR exclusively in the retrieval group, that was absent in low-performing animals and decreased again during LTR, as observed with BCN. However, with our approach and sampling size TNR did not change significantly in any of the training groups. In the cellular fraction we found a nonsignificant tendency toward a decrease in TNR abundance in all initial training sessions (Fig. 4A,C). In the hippocampus a comparable trend of decreased TNR abundance without significance was found for both, pellet and supernatant TNR (Fig. 4B,D; Table 1C-E).

The described alterations of BCN levels during training are most likely explained by the individual association learning performance of an animal. To exclude that other aspects of the training procedure, as for instance the amount of foot shocks or relief of aversive reinforcement, impact on ECM regulation, we tested auditory cortex tissue in a control group with unpaired tone and foot shock presentation (see Materials and Methods). In this group we neither observed an upregulation of full-length $\mathrm{BCN}$ in the supernatant fraction, nor a downregulation of the cleaved fragment in the pellet fraction (Fig. 5B).

To further relate the observed regulation of $\mathrm{BCN}$ and TNR to learning-derived alterations, rather than states of arousal or stress, changes of protein abundance were correlated with behavioral parameters. As the observed regulation of $\mathrm{BCN}$ and TNR was found to be transient during initial learning and recovered during LTR, we excluded data from the LTR group for correlation analysis. Full-length $\mathrm{BCN}$ in the soluble fraction positively correlated with the performance of an animal measured by the $d^{\prime}$ of its last session (Fig. $6 A$; correlation: $0.378 ; p=0.036$ ). Reaction times generally decrease with practice and performance (Logan, 1992). Correspondingly, a negative correlation was observed be-

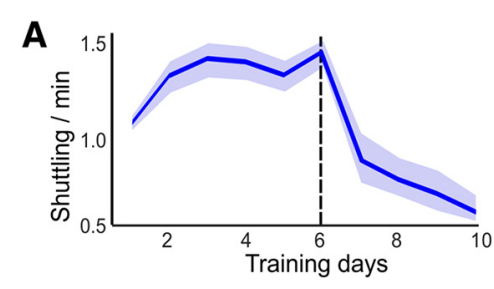

\section{B}


Pellet ACX
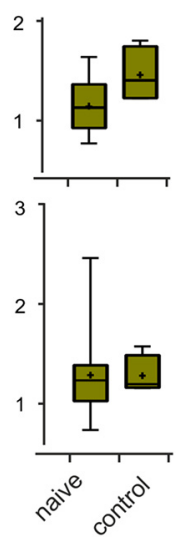

Figure 5. Unpaired tone and foot shock presentation did not change BCN levels in auditory cortex. $A$, In the control group $(n=4)$, we presented the same amount of foot shocks and frequency modulated sounds as in the retrieval group (average number of 180 foot shocks/animal). To mimic the relief of arousal in the later phase of training due to increase in performance, we presented foot shocks in the control group only within the first six sessions. Correspondingly, spontaneous compartment changes (escape shuttlings are subtracted), as a measure of arousal (Nienhuis and Olds, 1978), were high during the first six sessions, where animals received foot shocks ( $>1.0 / \mathrm{min})$. Dropped shuttling rates in the last four sessions indicate relief of the stress induced by the unpredictable foot shock presentation. $\boldsymbol{B}$, Quantification of Western blot signal intensity of $145 \mathrm{kDa} B C N, 55 \mathrm{kDa} B C N$, and TNR in the cellular (top) and extracellular fraction (bottom) of control animals. We did not observe any significant regulation of $\mathrm{BCN}$ or TNR measures in the pseudo-trained control group. Box plots represent median (bar) and interquartile range, and bars represent full range of data. Plus within each bar represents mean value. 
tween reaction time and $145 \mathrm{kDa} \mathrm{BCN}$ (Fig. $6 B$; corr: -0.37 ; $p=0.041$ ). In contrast, $145 \mathrm{kDa} \mathrm{BCN}$ levels did neither correlate with the absolute number of shocks received over the training procedure nor with the relative ratio of intertrial shuttles, both indicative of the level of arousal of an animal (Nienhuis and Olds, 1978; Fig. $6 C, D)$. We did not observe any other significant correlation with either learningrelated or stress/arousal-related parameters with any other $\mathrm{BCN}$ or TNR measure ( $p>0.05$; data not shown). TNR from the supernatant, but not the pellet fraction again showed a trend of positive correlation with the mean $d^{\prime}$ of the last session (Fig. 6E). In agreement with the close correlation with the soluble $145 \mathrm{kDa} \mathrm{BCN}$, we found a strong correlation between the abundance of both extracellular proteins (Fig. 6F).

Hence, cortex-dependent auditory learning yielded a performance-dependent transient upregulation of full-length BCN during the consolidation phase. This regulation of $\mathrm{BCN}$ was region-specific for the auditory cortex. Full-length TNR showed a comparable, but less prominent upregulation after successful learning. In addition, associative training led to a general downregulation of cleaved $\mathrm{BCN}$ in the auditory cortex and the hippocampus independent of individual performance, which was absent in the control group presented with unpaired tones and foot shocks (compare Fig. 5).

\section{Downregulation of full-length proteins} is specific for $\mathrm{BCN}$ in auditory cortex In a next step, we estimated the total change of ECM-associated BCN and TNR within auditory cortex and hippocampus by summing up the abundance of proteins from the soluble and pellet fraction in each training group (Fig. $7 A, B$ ). The total amount of $55 \mathrm{kDa}$ BCN showed a highly significant reduction in all training groups before the long-term retention interval in both, the auditory cortex and the hippocampus. Levels of cleaved BCN recovered during LTR (Table $1 F$ ). Fulllength $\mathrm{BCN}$ was only significantly modulated in the auditory cortex, but not the hippocampus. During early avoidance learning levels of full-length BCN were significantly lower compared with the naive and LTR group. Also LP animals showed significantly less abundance of $145 \mathrm{kDa} \mathrm{BCN}$ compared with these groups (Table $1 F$ ). During successful acquisition and retrieval, the total amount of full-length $\mathrm{BCN}$ was restored. However, restoration of $\mathrm{BCN}$ was not observed in animals that failed to learn the task, suggesting a performance-dependent regulation. In animals that were tested for long-term retrieval, BCN levels recovered back to basal levels (Fig. $7 A$, middle). In the hippocampus, total amounts of full-length BCN remained largely unaffected.
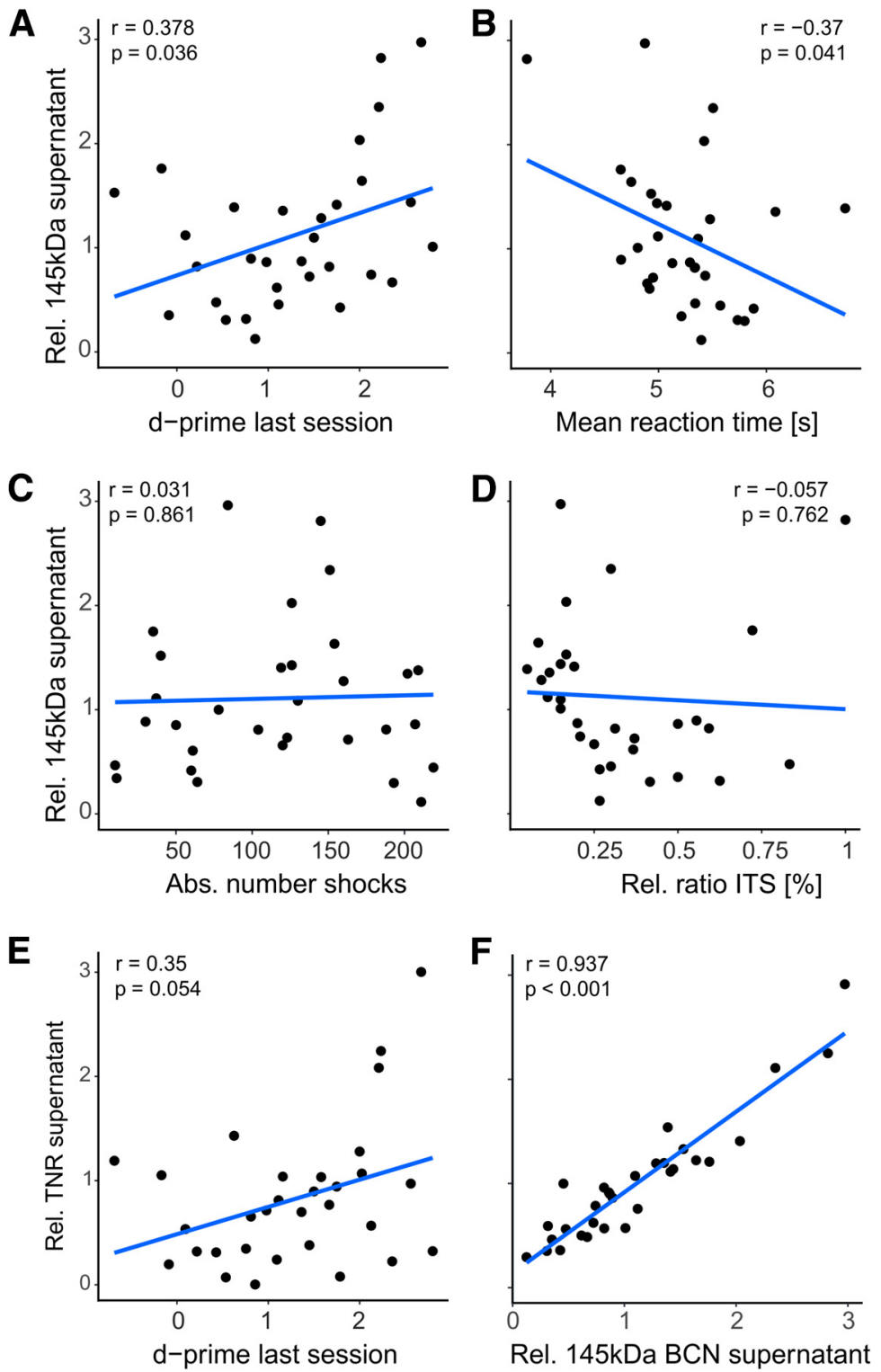

Figure 6. Correlation analysis of behavioral parameters and biochemical modulation of the $145 \mathrm{kDa} B C N$ fragment in the supernatant fraction. Data were taken from training groups $A V, A Q, R T$, and $L P$. $A$, Relative abundance of $145 \mathrm{kDa} B C N$ in the supernatant fraction was positively correlated with the $d^{\prime}$ value in the last training session indicating its performance dependent significant correlation was found between supernatant $145 \mathrm{kDa} B C N$ and absolute number of shocks $(C)$ or the ratio of relative intertrial shuttles $(\boldsymbol{D})$. $\boldsymbol{E}$, Relative abundance of TNR in the supernatant fraction showed a trend of a positive correlation with the $d^{\prime}$ the last training session $(p=0.054)$. Hence, this trend is in line with the performance-dependent upregulation of $145 \mathrm{BCN}$ in $\boldsymbol{A}$. $\boldsymbol{F}$, Furthermore, Pearson correlation of the both full-length proteins TNR and $145 \mathrm{kDa} B C N$ in the supernatant fraction were highly correlated. This implies a close interaction of the two ECM full-length proteins in the supernatant fraction and their differential regulation in the loose and cell-bound/PNN-associated ECM.

TNR showed only minimal reduction in the hippocampus that was most prominent during LTR (Table $1 G$ ). Total amount of TNR in the auditory cortex did not show the learning-related modulation as described for BCN.

\section{Discussion}

We demonstrate a performance-dependent regulation of the ECM-protein BCN in the sensory cortex during auditory learning and memory recall. Total amounts of full-length BCN and its $55 \mathrm{kDa}$ proteolytic fragment were decreased during initial training in the auditory cortex and hippocampus. Successful learning yielded a transient upregulation of full-length BCN specifically in 
A



Total amount TNR
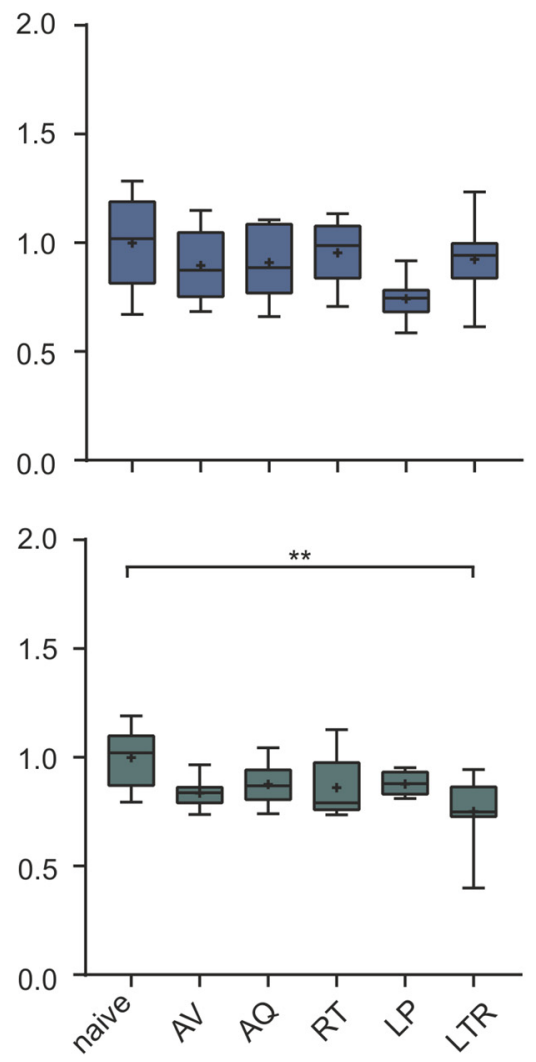

Figure 7. Changes of total levels of BCN and TNR in the auditory cortex and hippocampus along learning stages. $A$, Total amount of the $55 \mathrm{kDa} B C N$ (left), full-length $B C N$ (middle), and TNR (right) in auditory cortex measured as the sum of both supernatant and pellet fractions. Whereas total TNR showed no significant reduction in the auditory cortex, cleaved BCN showed a significant reduction across all training stages and recovered during LTR. Full-length BCN was also significantly reduced during early avoidance learning and also within the group of $L P$ animals. $B$, Total amount of the $55 \mathrm{kDa}$ BCN (left), full-length BCN (middle) and TNR (right) in hippocampus showed only generally reduced levels of the $55 \mathrm{kDa}$ BCN fragment independent of learning stage. For details, see Materials and Methods. *Indicates significant one-way ANOVA and post hoc Tukey tests of multiple comparisons between groups (Table $1 F$, G). Box plots represent median (bar) and interquartile range, and bars represent full range of data. Plus within each bar represents mean value.

the auditory cortex during consolidation not observed in animals that failed in discrimination. TNR, a binding partner of BCN, showed comparable, but less pronounced effects. During remote recall, BCN levels did not differ from naive animals. Together, this indicates a bimodal and complementary regulation of $\mathrm{BCN}$ during learning in a region-specific manner. The initial and experience-dependent downregulation of ECM proteins may provide a general state of elevated plasticity. Subsequently, learning-induced transient upregulation of fulllength BCN specifically in auditory cortex during retrieval is necessary for successful consolidation of recently learned memories (Fig. 8).

\section{Separation of loose ECM components from PNN-based and membrane-bound ECM}

The three forms of the hyaluronic-acid based ECM in the mature brain differ in their solubility (Celio et al., 1998; Deepa et al., 2006; Hedstrom et al., 2007; Sorg et al., 2016). Chondroitinase $\mathrm{ABC}$ extracts the loose ECM into the supernatant, whereas the PNN- and membrane-bound ECM remains in the cellular pellet fraction (Deepa et al., 2006; Frischknecht et al., 2009; Saroja et al., 2014). BCN, in particular, is not only present within the PNNs, but also highly abundant within the neuropil (Seidenbecher et al., 2002; Deepa et al., 2006; Ajmo et al., 2008; Valenzuela et al., 2014). Hence, changes in the soluble fraction can be assigned to the loose non-PNN ECM within the neuropil of the cortex, while changes in the pellet fraction reflect regulation of the cell-bound ECM and PNNs. Extracellular full-length BCN was upregulated exclusively in the retrieval phase but not in any other group (Fig. $3 C$; Table $1 D$ ). This suggests a learning-induced and transient upregulation of $\mathrm{BCN}$ within the neuropil. Hence, $\mathrm{BCN}$ regulation within the neuropil varies compared with $\mathrm{PNN} /$ cell surface $\mathrm{BCN}$, which demonstrates a differential ECM remodeling during learning dependent on learning stage, brain region, and cellular compartment.

\section{Differential regulation of cleaved and full-length BCN in auditory cortex}

BCN crosslinks ECM components by binding hyaluronic acid via its N-terminal domain and other ECM molecules via the C-terminus, as for instance TNR (Frischknecht and Seidenbecher, 2012). Cleavage of full-length BCN by proteases of the ADAMTS family, namely ADAMTS-4 and ADAMTS-5 (Tortorella et al., 1999, 2001; Nakamura et al., 2000), yields an $\mathrm{N}$-terminal fragment of $\sim 55 \mathrm{kDa}$ and separates the two functional domains (Frischknecht and Seidenbecher, 2012). Therefore, cleavage loosens the structure and density of the ECM, whereas the full-length protein stabilizes the ECM and increases its density. Previous data would suggest to find increased proteolytic cleavage during learning because extracellular proteolysis has been shown to support synaptic as well as structural plasticity (Dityatev et al., 2010). However, the $55 \mathrm{kDa}$ proteolytic fragment 

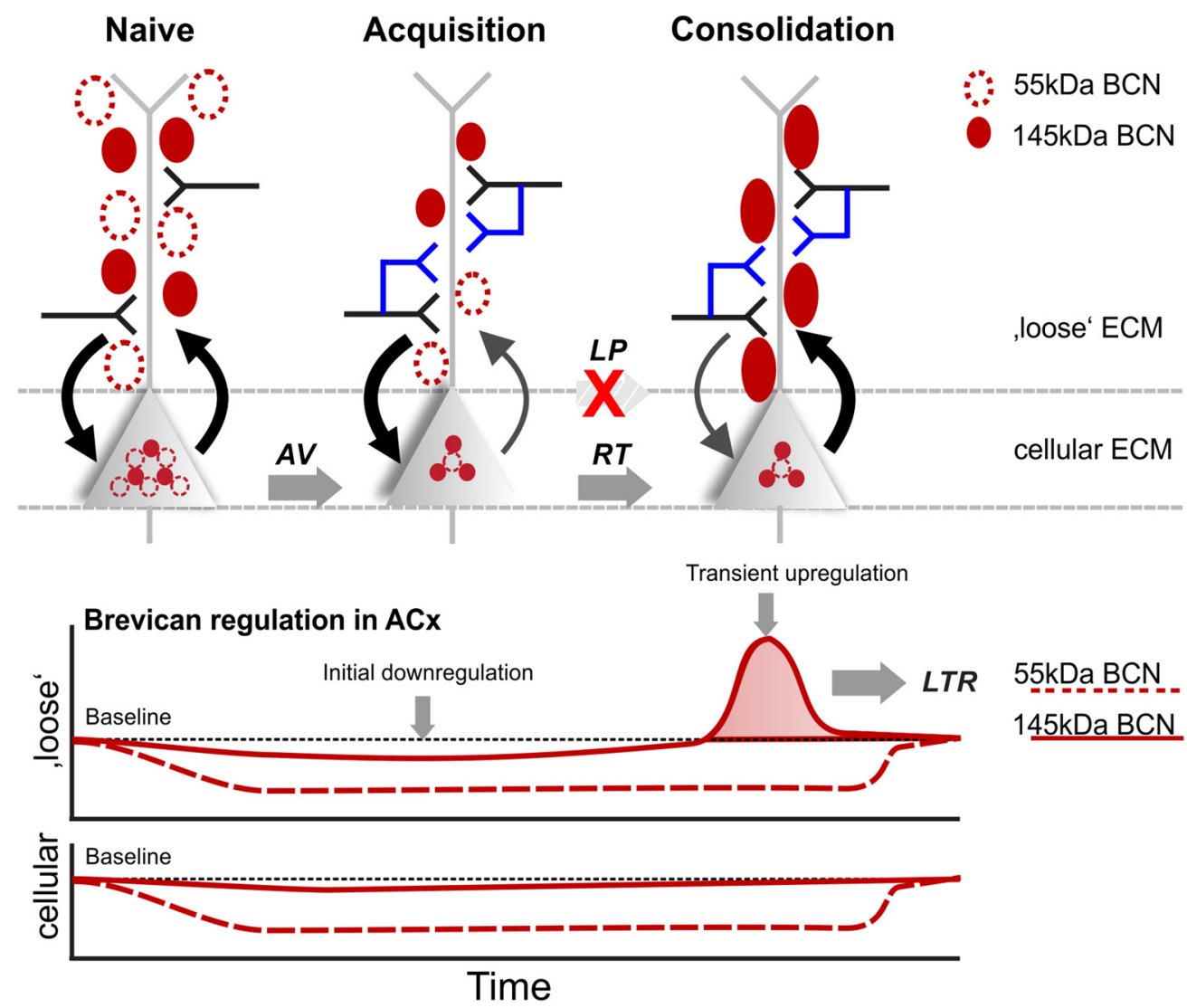

Figure 8. Learning-dependent regulation of $\mathrm{BCN}$ in auditory cortex. During acquisition of learning an initial downregulation of $\mathrm{BCN}$ in both, the cellular and loose $\mathrm{ECM}$ fraction (bottom) is permissive for learning-dependent synaptic remodeling (top). The cellular ECM here refers to the cell-bound and PNN-associated forms of the ECM, as extracted in the cellular fraction (pellet) of auditory cortex samples (Deepa et al., 2006). Here, new synaptic contacts are established during acquisition learning. Note that such downregulation was not present in the non-associative control group (Fig. 5), and hence, the downregulation is specific for early associative training. During consolidation of recent memories, a transient upregulation of the supernatant $145 \mathrm{kDa}$ full-length BCN (bottom) is obstructive for further structural plasticity. During this RT phase, recent memories are resistant against remodeling (Happel et al., 2014). This transient protection of recently acquired memories might be fundamental for their remote recall, as this was not found in LP animals. During LTR, levels of both BCN fragments returned to baseline, as no new (re-)learning is initiated.

of BCN was less abundant in trained animals compared with naive controls regardless of the brain region (Fig. $3 C, D$ ). Our results therefore suggest that the proteolytic fragment is specifically degraded soon after training Thus, cleavage is most likely the initial step in BCN degradation resulting in its net downregulation. Post-training sample collection $(>24 \mathrm{~h}$ after initial training) may miss the immediate increase of the $\mathrm{BCN}$ fragment in vivo because of proteolytic cleavage. Most studies describing increased abundance of proteolytic fragments of ECM proteins were performed in in vitro acutely after strong synaptic stimulation. For instance levels of proteolytic fragments of agrin were elevated after chemical LTP for only 15-20 min suggesting its fast removal (Matsumoto-Miyai et al., 2009). These proteolytic fragments have signaling functions and can promote the formation of dendritic protrusions. Fast removal of the fragment guarantees local and temporally limited signaling. However, whether a similar mechanism applies to the proteolytic fragment of $\mathrm{BCN}$ is a hypothesis that needs further investigation.

Controversial findings about learning-induced $\mathrm{BCN}$ regulation came from studies investigating spatial training in a Morris water maze. A recent study described downregulation during learning and recovery to basal levels after accomplishment (Favuzzi et al., 2017) in agreement with the downregulation of total proteolytic fragment and full-length protein, which recovered during remote recall in our study (Fig. 7). In contrast, Saroja et al.
(2014) found increased levels of hippocampal BCN and versican in the membrane fraction during memory recall. In agreement, we showed a transient and performance-dependent upregulation in soluble full-length BCN during the consolidation phase (Fig. 3C). Whereas Saroja et al. (2014) used a similar chondroitinase ABC-based ECM extraction method, Favuzzi et al. (2017) analyzed cell lysates, which correlates best with our PNN-enriched and cell-bound cellular fraction. Thus, taking into account the different extraction methods, both studies are in line with our findings. Downregulation of ECM proteins may be a general mechanism during task experience and engagement dependent on neither individual performance nor learning-related brain region. In contrast, the transient upregulation of full-length $\mathrm{BCN}$ was limited exclusively to the retrieval phase and restricted to the task-specific region within our cortex-dependent auditory paradigm. Elevated full-length $\mathrm{BCN}$ may support the consolidation process by restoring density and inhibitory properties of the ECM after the acquisition of the learned memory. Long-term retrieval did not induce regulation of BCN or TNR, indicating different cellular mechanisms or activity levels within the auditory cortex during initial learning compared with remote recall. Also, parameters not related to learning, but arousal did not reveal any regulation of ECM proteins in auditory cortex (Figs. 5, 6). 


\section{Learning and memory consolidation require reversed regulation of the ECM}

A recent report showed that $\mathrm{BCN}$ mRNA levels are upregulated within the auditory cortex during auditory fear conditioning, which is necessary for the expression of fear memory (Banerjee et al., 2017). In line with this, the region-specific and transient upregulation of BCN in our study may stabilize learning-induced neuronal networks and thus contribute to consolidation and memory formation. Our data now demonstrates that the time course of those processes differs between more demanding, cortex-dependent discrimination learning compared with Pavlovian fear conditioning (Banerjee et al., 2017). Correspondingly, we found a dynamic and success-dependent regulation of $\mathrm{BCN}$ over the course of 10 training days, and not only over several hours. Regulation of BCN protein can be achieved on different levels. Successful association learning may lead to decreased cleavage and subsequent degradation of BCN. Alternatively, learning may trigger $\mathrm{BCN}$ production and secretion leading to recovery of the extracellular full-length protein pools, while $\mathrm{BCN}$ processing may remain unaltered. The latter is supported by both our findings of higher amounts of full-length BCN in the soluble fraction exclusively during the consolidation phase and by recent findings of elevated BCN mRNA levels after fear conditioning (Banerjee et al., 2017). This upregulation was only transient. Neither low performing animals nor animals during LTR showed differences in any of the BCN measures (Figs. 3, 6). Together, our results therefore indicate that the immediate increase in $\mathrm{BCN}$ cleavage during early training may promote learning-dependent plasticity. Second, successful learning led to the production and secretion of de novo synthesized $\mathrm{BCN}$. This reconstitution restores the downregulated ECM during consolidation in the service of protecting the recently established memory over a transient period of time. Most importantly, only subjects that have learned the discrimination task showed such bimodal regulation of a more flexible or rigid ECM state during learning and memory consolidation, respectively (Fig. 8).

Although transcripts for soluble and membrane-bound BCN have been related to glial cells and PV interneurons, respectively (Favuzzi et al., 2017), the cellular origin of the bimodal regulation is still elusive. Nevertheless, dopamine released in auditory cortex during acquisition learning (cf. Happel, 2016) may regulate PV cells during learning and memory formation (Karunakaran et al., 2016). Further, ECM removal promotes short-term-dependent plasticity because of increased AMPA-receptor motility (Heine et al., 2008; Frischknecht et al., 2009). Both mechanisms may interact during states of downregulated ECM to tune labile synaptic contacts during early learning (Hofer and Bonhoeffer, 2010; Mongillo et al., 2017). To protect recently established memories, a compensatory transient upregulation of the ECM would limit the permissive impact on plasticity. Our data further shows that such protection is restricted to the consolidation of recent, not of remote memories, as protein levels are back to naive levels during LTR. In this line, enzymatic degradation of the ECM has only subtle effects on several forms of initial learning (Paylor et al., 2018), but interferes with the extinction or reversal of recently established memories (Gogolla et al., 2009; Happel et al., 2014). The complex molecular orchestra of cellular and extracellular signals may hence first promote plastic reorganizations and subsequently support their learning-dependent fixation (Donato et al., 2013, Kamigaki and Dan, 2017; Ognjanovski et al., 2017). Together, we revealed a temporal and region-specific bimodal regulation of $\mathrm{BCN}$ promoting acquisition of learning and remote memory resistance via supported consolidation (Fig. 8).

\section{References}

Ajmo JM, Eakin AK, Hamel MG, Gottschall PE (2008) Discordant localization of WFA reactivity and brevican/ADAMTS-derived fragment in rodent brain. BMC Neurosci 9:14.

Banerjee SB, Gutzeit VA, Baman J, Aoued HS, Doshi NK, Liu RC, Ressler KJ (2017) Perineuronal nets in the adult sensory cortex are necessary for fear learning article perineuronal nets in the adult sensory cortex are necessary for fear learning. Neuron 95:169-179.e3.

Bozzelli PL, Alaiyed S, Kim E, Villapol S, Conant K (2018) Proteolytic remodeling of perineuronal nets: effects on synaptic plasticity and neuronal population dynamics. Neural Plast 2018:5735789.

Celio MR, Spreafico R, De Biasi S, Vitellaro-Zuccarello L (1998) Perineuronal nets: past and present. Trends Neurosci 21:510-515.

Deepa SS, Carulli D, Galtrey C, Rhodes K, Fukuda J, Mikami T, Sugahara K, Fawcett JW (2006) Composition of perineuronal net extracellular matrix in rat brain: a different disaccharide composition for the netassociated proteoglycans. J Biol Chem 281:17789-17800.

Deliano M, Scheich H, Ohl FW (2009) Auditory cortical activity after intracortical microstimulation and its role for sensory processing and learning. J Neurosci 29:15898-15909.

de Vivo L, Landi S, Panniello M, Baroncelli L, Chierzi S, Mariotti L, Spolidoro M, Pizzorusso T, Maffei L, Ratto GM (2013) Extracellular matrix inhibits structural and functional plasticity of dendritic spines in the adult visual cortex. Nat Commun 4:1484.

Dityatev A, Schachner M, Sonderegger P (2010) The dual role of the extracellular matrix in synaptic plasticity and homeostasis. Nat Rev Neurosci 11:735-746.

Donato F, Rompani SB, Caroni P (2013) Parvalbumin-expressing basketcell network plasticity induced by experience regulates adult learning. Nature 504:272-276.

Dudai Y (2012) The restless engram: consolidations never end. Annu Rev Neurosci 35:227-247.

Favuzzi E, Marques-Smith A, Deogracias R, Winterflood CM, SánchezAguilera A, Mantoan L, Maeso P, Fernandes C, Ewers H, Rico B (2017) Activity-dependent gating of parvalbumin interneuron function by the perineuronal net protein brevican. Neuron 95:639-655.e10.

Frischknecht R, Seidenbecher CI (2012) Brevican: a key proteoglycan in the perisynaptic extracellular matrix of the brain. Int J Biochem Cell Biol 44:1051-1054.

Frischknecht R, Fejtova A, Viesti M, Stephan A, Sonderegger P (2008) Activity-induced synaptic capture and exocytosis of the neuronal serine protease neurotrypsin. J Neurosci 28:1568-1579.

Frischknecht R, Heine M, Perrais D, Seidenbecher CI, Choquet D, Gundelfinger ED (2009) Brain extracellular matrix affects AMPA receptor lateral mobility and short-term synaptic plasticity. Nat Neurosci 12:897904.

Gogolla N, Caroni P, Lüthi A, Herry C (2009) Perineuronal nets protect fear memories from erasure. Science 325:1258-1261.

Happel MF (2016) Dopaminergic impact on local and global cortical circuit processing during learning. Behav Brain Res 299:32-41.

Happel MF, Niekisch H, Castiblanco Rivera LL, Ohl FW, Deliano M, Frischknecht R (2014) Enhanced cognitive flexibility in reversal learning induced by removal of the extracellular matrix in auditory cortex. Proc Natl Acad Sci U S A 111:2800-2805.

Happel MFK, Deliano M, Ohl FW (2015) Combined shuttle-box training with electrophysiological cortex recording and stimulation as a tool to study perception and learning. J Vis Exp 104:e53002.

Hedstrom KL, Xu X, Ogawa Y, Frischknecht R, Seidenbecher CI, Shrager P, Rasband MN (2007) Neurofascin assembles a specialized extracellular matrix at the axon initial segment. J Cell Biol 178:875-886.

Heine M, Groc L, Frischknecht R, Béique JC, Lounis B, Rumbaugh G, Huganir RL, Cognet L, Choquet D (2008) Surface mobility of postsynaptic AMPARs tunes synaptic transmission. Science 320:201-205.

Hofer SB, Bonhoeffer T (2010) Dendritic spines: the stuff that memories are made of? Curr Biol 20:R157-R159.

Hylin MJ, Orsi SA, Moore AN, Dash PK (2013) Disruption of the perineuronal net in the hippocampus or medial prefrontal cortex impairs fear conditioning. Learn Mem 20:267-273.

Kähne T, Kolodziej A, Smalla KH, Eisenschmidt E, Haus UU, Weismantel R, Kropf S, Wetzel W, Ohl FW, Tischmeyer W, Naumann M, Gundelfinger ED (2012) Synaptic proteome changes in mouse brain regions upon auditory discrimination learning. Proteomics 12:2433-2444. 
Kamigaki T, Dan Y (2017) Delay activity of specific prefrontal interneuron subtypes modulates memory-guided behavior. Nat Neurosci 20:854863.

Karunakaran S, Chowdhury A, Donato F, Quairiaux C, Michel CM, Caroni P (2016) PV plasticity sustained through D1/5 dopamine signaling required for long-term memory consolidation. Nat Neurosci 19:454-464.

Ladner CL, Yang J, Turner RJ, Edwards RA (2004) Visible fluorescent detection of proteins in polyacrylamide gels without staining. Anal Biochem 326:13-20.

Logan GD (1992) Shapes of reaction-time distributions and shapes of learning curves: a test of the instance theory of automaticity. J Exp Psychol Learn Mem Cogn 18:883-914.

Matsumoto-Miyai K, Sokolowska E, Zurlinden A, Gee CE, Lüscher D, Hettwer S, Wölfel J, Ladner AP, Ster J, Gerber U, Rülicke T, Kunz B, Sonderegger P (2009) Coincident pre- and postsynaptic activation induces dendritic filopodia via neurotrypsin-dependent agrin cleavage. Cell 136:1161-1171.

Mongillo G, Rumpel S, Loewenstein Y (2017) Intrinsic volatility of synaptic connections: a challenge to the synaptic trace theory of memory. Curr Opin Neurobiol 46:7-13.

Morellini F, Sivukhina E, Stoenica L, Oulianova E, Bukalo O, Jakovcevski I, Dityatev A, Irintchev A, Schachner M (2010) Improved reversal learning and working memory and enhanced reactivity to novelty in mice with enhanced GABAergic innervation in the dentate gyrus. Cereb Cortex 20:2712-2727.

Nakamura H, Fujii Y, Inoki I, Sugimoto K, Tanzawa K, Matsuki H, Miura R, Yamaguchi Y, Okada Y (2000) Brevican is degraded by matrix metalloproteinases and aggrecanase-1 (ADAMTS4) at different sites. J Biol Chem 275:38885-38890.

Nienhuis R, Olds J (1978) Changes in unit responses to tones after food reinforcement in the auditory pathway of the rat: intertrial arousal. Exp Neurol 59:229-242.

Ognjanovski N, Schaeffer S, Wu J, Mofakham S, Maruyama D, Zochowski M, Aton SJ (2017) Parvalbumin-expressing interneurons coordinate hippocampal network dynamics required for memory consolidation. Nat Commun 8:15039.

Ohl FW, Scheich H (2005) Learning-induced plasticity in animal and human auditory cortex. Curr Opin Neurobiol 15:470-477.

Ohl FW, Wetzel W, Wagner T, Rech A, Scheich H (1999) Bilateral ablation of auditory cortex in Mongolian gerbil affects discrimination of frequency modulated tones but not of pure tones. Learn Mem 6:347-362.

Paylor JW, Wendlandt E, Freeman TS, Greba Q, Marks WN, Howland JG, Winship IR (2018) Impaired cognitive function after perineuronal net degradation in the medial prefrontal cortex. Eneuro 5:ENEURO.025318.2018 .

Pizzorusso T, Medini P, Berardi N, Chierzi S, Fawcett JW, Maffei L (2002) Reactivation of ocular dominance plasticity in the adult visual cortex. Science 298:1248-1251.
Reichenbach N, Herrmann U, Kähne T, Schicknick H, Pielot R, Naumann M, Dieterich DC, Gundelfinger ED, Smalla KH, Tischmeyer W (2015) Differential effects of dopamine signalling on long-term memory formation and consolidation in rodent brain. Proteome Sci 13:13.

Romberg C, Yang S, Melani R, Andrews MR, Horner AE, Spillantini MG, Bussey TJ, Fawcett JW, Pizzorusso T, Saksida LM (2013) Depletion of perineuronal nets enhances recognition memory and long-term depression in the perirhinal cortex. J Neurosci 33:7057-7065.

Saroja SR, Sase A, Kircher SG, Wan J, Berger J, Höger H, Pollak A, Lubec G (2014) Hippocampal proteoglycans brevican and versican are linked to spatial memory of Sprague-Dawley rats in the Morris water maze. J Neurochem 130:797-804.

Seidenbecher CI, Smalla KH, Fischer N, Gundelfinger ED, Kreutz MR (2002) Brevican isoforms associate with neural membranes. J Neurochem 83:738-746.

Sorg BA, Berretta XS, Blacktop XJ, Fawcett XJ, Kitagawa XH, Kwok XJ, Miquel XM (2016) Casting a wide net: role of perineuronal nets in neural plasticity. J Neurosci 36:11459-11468.

Stark H, Scheich H (1997) Dopaminergic and serotonergic neurotransmission systems are differentially involved in auditory cortex learning: a longterm microdialysis study of metabolites. J Neurochem 68:691-697.

Tortorella MD, Burn TC, Pratta MA, Abbaszade I, Hollis JM, Liu R, Rosenfeld SA, Copeland RA, Decicco CP, Wynn R, Rockwell A, Yang F, Duke JL, Solomon K, George H, Bruckner R, Nagase H, Itoh Y, Ellis DM, Ross H, et al. (1999) Purification and cloning of aggrecanase-1: a member of the ADAMTS family of proteins. Science 284:1664-1666.

Tortorella MD, Malfait AM, Deccico C, Arner E (2001) The role of ADAMTS4 (aggrecanase-1) and ADAM-TS5 (aggrecanase-2) in a model of cartilage degradation. Osteoarthr Cartil 9:539-552.

Valenzuela J, Christopher H, Gilbert F, Jeet S, Barbara S, Constanze S, Frischknecht (2014) Hyaluronan-based extracellular matrix under conditions of homeostatic plasticity. Philos Trans R Soc Lond B Biol Sci 369: 20130606

Végh MJ, Heldring CM, Kamphuis W, Hijazi S, Timmerman AJ, Li KW, van Nierop P, Mansvelder HD, Hol EM, Smit AB, van Kesteren RE (2014) Reducing hippocampal extracellular matrix reverses early memory deficits in a mouse model of Alzheimer's disease. Acta Neuropathol Commun 2:76.

Wetzel W, Wagner T, Ohl FW, Scheich H (1998) Categorical discrimination of direction in frequency-modulated tones by Mongolian gerbils. Behav Brain Res 91:29-39.

Xue YX, Xue LF, Liu JF, He J, Deng JH, Sun SC, Han HB, Luo YX, Xu LZ, Wu $\mathrm{P}, \mathrm{Lu} \mathrm{L}$ (2014) Depletion of perineuronal nets in the amygdala to enhance the erasure of drug memories. J Neurosci 34:6647-6658.

Yang S, Cacquevel M, Saksida LM, Bussey TJ, Schneider BL, Aebischer P, Melani R, Pizzorusso T, Fawcett JW, Spillantini MG (2015) Perineuronal net digestion with chondroitinase restores memory in mice with tau pathology. Exp Neurol 265:48-58. 